\title{
REDUCIBILITY OF SOME GENERALIZED PRINCIPAL SERIES OF THE METAPLECTIC GROUP
}

\author{
IgOR CigAnOvić \\ University of Zagreb, Croatia
}

\begin{abstract}
We determine reducibility of the representation of the metaplectic group induced from the tensor product of an essentially square integrable representation attached to the Zelevinsky segment and a genuine cuspidal representation of the metaplectic group.
\end{abstract}

\section{INTRODUCTION}

Let $F$ be a non-Archimedean local field of the characteristic zero. The metaplectic group $S p(n, F)$ is the unique non-trivial two-fold central extension of the symplectic group $S p(n, F)$. Even though it is not a linear algebraic group, a number of representation theoretic results have been expanded. Notably, the extension of the theory of Bernstein and Zelevinsky ([1], [2], [21]) can be seen in [8] and [10]. Since $G L(n, F)$ can be embeded into $\operatorname{Sp}(n, F)$, we denote by $\widehat{G L(n, F)}$ its two-fold cover. Given a non-trivial additive character $\psi$ of $F$, there exists a genuine character $\chi_{\psi}$ of $\widetilde{G(n, F)}$ corresponding to it. The mapping $\pi \mapsto \chi_{\psi} \pi$ sets up a bijection between irreducible representations of $G L(n, F)$ and genuine irreducible representations of $\widetilde{G(n, F)}$ (see section 4.1 of $[8])$.

Let ||$_{F}$ be the normalized absolute value of $F, \nu=|\operatorname{det}|_{F}$ and $\rho$ a unitary irreducible cuspidal representation of $G L(n, F)$. Let $a, b$ be real numbers, such that $a+b+1 \in \mathbb{Z}_{>0}$. The set $\Delta=\left\{\chi_{\psi} \nu^{-b} \rho, \ldots, \chi_{\psi} \nu^{a} \rho\right\}$ is called

2010 Mathematics Subject Classification. 22D12, 22E50, 22D30, 11F85.

Key words and phrases. Metaplectic group, $p$-adic field, parabolic induction, Jacquet module, generalized principal series.

This work has been fully supported by Croatian Science Foundation under the project 9364 . 
a segment. To the segment $\Delta$ is attached a genuine irreducible essentially square integrable representation $\delta(\Delta) \hookrightarrow \chi_{\psi} \nu^{a} \rho \times \cdots \times \chi_{\psi} \nu^{-b} \rho$, denoted by $\delta\left(-b, a, \chi_{\psi} \rho\right)$. Let now $\sigma$ be a genuine irreducible cuspidal representation of the metaplectic group. We are considering reducibility of the induced representation $\delta\left(-b, a, \chi_{\psi} \rho\right) \rtimes \sigma=\operatorname{Ind} \widetilde{S \widetilde{S(n, F)}}\left(\delta\left(-b, a, \chi_{\psi} \rho\right) \otimes \sigma\right)$ for the appropriate Levi subgroup $\widetilde{M_{s}}$ of $\widetilde{S p(n, F)}$ (see (2.2) for the notation). More precisely, we prove the following theorem.

TheOREM 1.1. Let $a, b$ be real numbers, such that $a+b+1 \in \mathbb{Z}_{>0}, \rho a$ unitary irreducible cuspidal representation of $G L(n, F)$ and $\sigma$ a genuine irreducible cuspidal representation of the metaplectic group. The representation $\delta\left(-b, a, \chi_{\psi} \rho\right) \rtimes \sigma$ reduces if and only if there exists a real number $-b \leq s \leq a$, such that $a-s$ is an integer and $\chi_{\psi} \nu^{s} \rho \rtimes \sigma$ reduces.

This result was proved for classical groups in [19], Theorem 13.2, assuming the cuspidal reducibility at $\frac{1}{2} \mathbb{Z}$ i.e. let $\rho$ be an irreducible unitary cuspidal representation of the general linear group and $\sigma$ a similar representation of the symplectic or the odd split special orthogonal group. If $\rho$ is self-contragredient then there exists $\alpha_{0} \in(1 / 2) \mathbb{Z}$ such that $\nu^{\alpha} \rho \rtimes \sigma$ is irreducible for $\alpha \in \mathbb{R} \backslash\left\{ \pm \alpha_{0}\right\}$ and $\nu^{\alpha_{0}} \rho \rtimes \sigma$ and $\nu^{-\alpha_{0}} \rho \rtimes \sigma$ reduce. If $\rho$ is not self-contragredient then $\nu^{\alpha} \rho \rtimes \sigma$ is irreducible for all $\alpha \in \mathbb{R}$. This statement is provided by the recent work of Mœglin, Theorem 3.1.1 of [15], and the metaplectic version follows from results of Hanzer and Muić ([9]), which relate the cuspidal reducibility for the odd special orthogonal and the metaplectic group. For more details see Proposition 3.1.

Thus, we rework the proof of the above theorem for the metaplectic group, using as the starting point the cuspidal reducibility for the metaplectic group as well as tools of the parabolic induction and Jacquet modules ([8]).

\section{Preliminaries}

Let $F$ be a non-Archimedean local field of characteristic zero. For an integer $n \geq 1$, let $S p(n, F)$ be the group of $F$-points of the $F$-split symplectic group of rank $n$ defined over $F$. We use matrix realization as in section 1 of [10]. The metaplectic group $\widehat{S p(n, F)}$ is the unique, non-trivial, two-fold central extension of $\operatorname{Sp}(n, F)$. We have an exact sequence

$$
1 \longrightarrow \mu_{2} \stackrel{i}{\longrightarrow} \widehat{S p(n, F)} \stackrel{p}{\longrightarrow} S p(n, F) \longrightarrow 1,
$$

where $\mu_{2}=\{ \pm 1\}$ is the multiplicative group. As a set $\widetilde{S p(n, F)}=S p(n, F) \times$ $\mu_{2}$ and $p([g, \epsilon])=g$ for $g \in S p(n, F), \epsilon \in \mu_{2}$. Also, we have the two-fold central extension $G \widehat{L(n, F)}$ of $G L(n, F)$ given as the preimage with respect to $p$ of the embedding of $G L(n, F)$ into $S p(n, F)$. By convention, for $n=0$ 
all covering groups are considered to be $\mu_{2}$, and all linear groups to be the trivial group.

We fix the Borel subgroup as in section 1 of [10]. Let $m$ be an integer $0 \leq m \leq n$ and $\left(n_{1}, \ldots, n_{k}\right)$ an ordered partition of $m$ if $m>0$, or $\left(n_{1}\right)=(0)$ if $m=0$. Let $s=\left(n_{1}, \ldots, n_{k} ; n_{0}\right)$ where $n_{0}=n-\left(n_{1}+\cdots+n_{k}\right)$. Such $s$ parametrize standard parabolic subgroups $P_{s}$ of $S p(n, F)$. We write the Levi decomposition $P_{s}=M_{s} N_{s}$, where $M_{s}$ is the Levi factor and $N_{s}$ the unipotent radical. Here

$$
M_{s} \cong G L\left(n_{1}, F\right) \times \cdots \times G L\left(n_{k}, F\right) \times S p\left(n_{0}, F\right) .
$$

Let $\widetilde{P_{s}}$ and $\widetilde{M_{s}}$ be preimages of $P_{s}$ and $M_{s}$ in $\widetilde{S p(n, F)}$ with respect to the projection $p$, and $N_{s}^{\prime}=N_{s} \times\{1\}$. Then $\widetilde{P_{s}}$ are standard parabolic subgroups of $\widetilde{S p(n, F)}$, and there is a Levi decomposition $\widetilde{P_{s}}=\widetilde{M_{s}} N_{s}^{\prime}$. By page 60 of [17], we call $\widetilde{M_{s_{1}}}, s_{1}=\left(n_{1}^{(1)}, \ldots, n_{k_{1}}^{(1)} ; n_{0}^{(1)}\right)$ and $\widetilde{M_{s_{2}}}, s_{2}=\left(n_{1}^{(2)}, \ldots, n_{k_{2}}^{(2)} ; n_{0}^{(2)}\right)$ associate if $M_{s_{1}}$ and $M_{s_{2}}$ are associate, that is if there exists $w$ in the normalizer of $M_{(1, \ldots, 1 ; 0)}$ in $S p(n, F)$ such that $w M_{s_{1}} w^{-1}=M_{s_{2}}$. This is equivalent to $k_{1}=k_{2}$ and $\left(n_{1}^{(1)}, \ldots, n_{k_{1}}^{(1)}\right)$ and $\left(n_{1}^{(2)}, \ldots, n_{k_{2}}^{(2)}\right)$ are equal as unordered partitions of the same number (see page 9 of [20]).

There exists an epimorphism $\phi$ with finite kernel ([10], [8, p. 243])

$$
\widetilde{G\left(n_{1}, F\right)} \times \cdots \times \widehat{G\left(n_{k}, F\right)} \times \widehat{S p\left(n_{0}, F\right)} \stackrel{\phi}{\longrightarrow} \widetilde{M_{s}}
$$

given by

$$
\left(\left(g_{1}, \epsilon_{1}\right), \ldots,\left(g_{k}, \epsilon_{k}\right),(h, \epsilon)\right) \mapsto\left(\left(g_{1}, \ldots, g_{k}, h\right), \epsilon_{1} \cdots \epsilon_{k} \epsilon \alpha\right)
$$

where $\alpha=\prod_{i<j}\left(\operatorname{det} g_{i}, \operatorname{det} g_{j}\right)_{F} \cdot\left(\prod_{j=1}^{k}\left(\operatorname{det} g_{j}, x(h)\right)_{F}\right)$ and $x(h)$ is as in Lemma 5.1 of [18]. Similarly, fixing the Borel subgroup in $G L(n, F)$, the standard parabolic subgroups $P_{s}=M_{s} N_{s}$ of $G L(n, F)$ and $\widetilde{P_{s}}=\widetilde{M}_{s} N_{s}^{\prime}$, of $\widehat{G L(n, F)}$ are parameterized by ordered partitions $s=\left(n_{1}, \ldots, n_{k}\right)$ of $n$ into positive integers. We have $M_{s} \cong G L\left(n_{1}, F\right) \times \cdots \times G L\left(n_{k}, F\right)$ and there is an epimorphism with finite kernel

$$
\widetilde{G\left(n_{1}, F\right)} \times \cdots \times \widehat{G\left(n_{k}, F\right)} \stackrel{\phi}{\longrightarrow} \widetilde{M_{s}} \leq \widehat{G(n, F)} .
$$

Groups $\widetilde{S p(n, F)}$ and $\widetilde{(L(n, F)}$ are locally compact totally disconnected Hausdorff topological groups and we have usual notions of smooth and admissible representations as well as functors of normalized induction and Jacquet modules ([2], [17, p. 59]). Representations that do not act trivially by $\mu_{2}$ are called genuine. Let $\mathrm{Alg}$ denote the category of smooth representations, i.e. we consider complex representations such that the stabilizer of any vector is an open subgroup. Let $\widetilde{M}_{s} \leq \widehat{S p(n, F)}$ as above. We have the functor of the 
normalized parabolic induction

$$
\operatorname{Ind} \widetilde{S(n, F)}: \operatorname{Alg}\left(\widetilde{M}_{M_{s}}\right) \rightarrow \operatorname{Alg}(\widetilde{S p(n, F)})
$$

defined as follows. Since $N_{s}^{\prime}=N_{s} \times\{1\}$ is a closed subgroup of $\widehat{\operatorname{Sp(n,F)} \text { we }}$ identify Haar measures on $N_{s}^{\prime}$ and $N_{s}$. By section 3.3 of [8] for $\widetilde{m}=[m, \epsilon] \epsilon$ $\widetilde{M}_{s}$ and $n \in N_{s}$ we have $\widetilde{m}^{-1}[n, 1] \widetilde{m}=\left[m^{-1} n m, 1\right]$. Thus for topological modules $\delta_{N_{s}^{\prime}}$ and $\delta_{N_{s}}$ defined as in [2] p. 444.,

$$
\begin{gathered}
\int_{N_{s}^{\prime}} f\left(\widetilde{m}^{-1} n^{\prime} \tilde{m}\right) d \mu\left(n^{\prime}\right)=\delta_{N_{s}^{\prime}}(\widetilde{m}) \int_{N_{s}^{\prime}} f\left(n^{\prime}\right) d \mu\left(n^{\prime}\right), f \in L^{1}\left(N_{s}^{\prime}, \mu\right), \\
\int_{N_{s}} f\left(m^{-1} n m\right) d \mu(n)=\delta_{N_{s}}(m) \int_{N_{s}} f(n) d \mu(n), f \in L^{1}\left(N_{s}, \mu\right)
\end{gathered}
$$

we have $\delta_{N_{s}^{\prime}}(\widetilde{m})=\delta_{N_{s}}(m)$. Now suppose that $\rho \in \operatorname{Alg}\left(\widetilde{M}_{s}\right)$ acts on a complex vector space $L$. Let $V$ be the vector space of functions $f: \widetilde{S p(n, F)} \rightarrow L$ such that two conditions hold:

1. $\left.f\left(\widetilde{m} n^{\prime} \widetilde{g}\right)=\delta_{N_{s}}(m) \rho(\widetilde{m}) f(\widetilde{g}), \widetilde{m}=[m, \epsilon] \in \widetilde{M}_{s}, n^{\prime} \in N_{s}^{\prime} \widetilde{g} \in \widetilde{\operatorname{Sp(n,F}}\right)$.

2. There exists an open subgroup $K_{f} \subseteq \widehat{S p(n, F)}$ such that

$$
f(\widetilde{g} k)=f(\widetilde{g}), k \in K_{f}, \widetilde{g} \in \widetilde{S p(n, F)} .
$$

The induced representation $\operatorname{Ind}_{\widetilde{M_{s}}} \widetilde{S(n, F)}(\rho)$ acts on $V$ by the right translation:

$$
\left(\left[\left(\operatorname{Ind}_{\widetilde{M}_{s}} \widetilde{\widetilde{p}_{(n, F)}}(\rho)\right)(\widetilde{g})\right] f\right)\left(\widetilde{g}^{\prime}\right)=f\left(\widetilde{g} \widetilde{g}^{\prime}\right), \quad \widetilde{g}, \widetilde{g}^{\prime} \in \widetilde{S p(n, F)} .
$$

Now we define the Jacquet module functor:

$$
r_{s}=\operatorname{Jacq}_{s}=\operatorname{Jacq} \frac{\widetilde{S p(n, F)}}{\widetilde{M_{s}}}: \operatorname{Alg}(\widetilde{S p(n, F)}) \rightarrow \operatorname{Alg}\left(\widetilde{M}_{s}\right) .
$$

Suppose that $\sigma \in \operatorname{Alg}(\widetilde{S p(n, F)})$ acts on a complex vector space $V$. Let $V\left(N_{s}\right)$ be the subspace of $V$ spanned by the vectors of the form $\sigma\left(n^{\prime}\right) v-v, n^{\prime} \in$ $N^{\prime}, v \in V$. It is $\widetilde{M}_{s}$ invariant. The representation $\operatorname{Jacq}_{\widetilde{M}_{s}} \widetilde{S p(n, F)}(\sigma)$ acts on $V / V\left(N_{s}\right)$ as

$\left[\left(\operatorname{Jacq}_{\widetilde{M_{s}}} \widetilde{S p(n, F)}(\sigma)\right)(\widetilde{m})\right]\left(v+V\left(N_{s}\right)\right)=\delta_{N_{s}}^{-\frac{1}{2}}(\widetilde{m})\left(\sigma(\widetilde{m}) v+V\left(N_{s}\right)\right), \quad \widetilde{m} \in \widetilde{M}_{s}, v \in V$.

For $\sigma$ in $\operatorname{Alg}(\widetilde{S p(n, F)})$ and $\rho$ in $\operatorname{Alg}\left(\widetilde{M}_{s}\right)$, we have the Frobenius reciprocity (section 1.5 of $[10]$ )

$$
\operatorname{Hom}_{S p(n, F)}\left(\sigma, \operatorname{Ind}_{\widetilde{M_{s}}} \widetilde{S(n, F)}(\rho)\right) \cong \operatorname{Hom}_{\widetilde{M_{s}}}\left(\operatorname{Jacq}_{\frac{S p(n, F)}{M_{s}}}(\sigma), \rho\right) .
$$

Recall that $\sigma$ is a cuspidal representation of $\widetilde{S p(n, F)}$ if the Jacquet module of $\sigma$ with respect to any proper parabolic subgroup is trivial. Every irreducible 
representation can be embedded into a representation parabolically induced from a cuspidal one (section 1.5 of [10], Proposition 4.4 of [8]).

Fix a non-trivial additive character $\psi$ of $F$. The mapping $x \mapsto \psi\left(x^{2}\right)$ is a character of second degree on $F$ and we denote its Weil index with $\gamma(\psi)$. More details and properties can be found in the Appendix of [18]. For $a \in F^{\times}$, we let

$$
\gamma(a, \psi)=\gamma\left(\psi_{a}\right) \gamma(\psi)^{-1},
$$

where $\psi_{a}(x)=\psi(a x), x \in F$. Now

$$
\chi_{\psi}(g, \epsilon)=\epsilon \gamma\left(\operatorname{det} g, \psi_{\frac{1}{2}}\right)^{-1},
$$

where $g \in G L(n, F), \epsilon \in \mu_{2}$, defines a genuine character of $\left.\widetilde{G(n, F)}\right)([10$, p. 232]). The mapping

$$
L_{\psi}: \pi \mapsto \chi_{\psi} \pi
$$

sets up a bijection between $\operatorname{Alg}(G L(n, F))$ and the category of the smooth

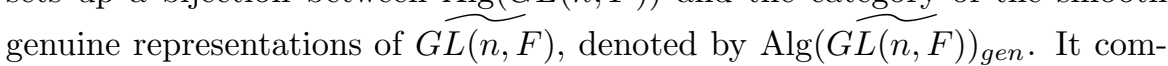
mutes with the parabolic induction and the Jacquet module (see Proposition 4.1 of [8]) preserving the length of representations. The genuine irreducible representation of $G(0, F)=\mu_{2}$ is written as $\chi_{\psi} \mathbf{1}$, where $\mathbf{1}$ denotes the irreducible representation of the trivial group. To see how the parametrization of $\operatorname{Alg}(G \widetilde{L(n, F)})_{\text {gen }}$ depends on the choice of $\psi$, let $\psi^{\prime}$ be another non-trivial additive character of $F$. There exists $a \in F^{\times}$such that $\psi^{\prime}=\psi_{a}$. Let $(,)_{F}$ be the Hilbert symbol of field $F$. By Corollary A.5 of [18] $\gamma\left(b, \psi_{a}\right)=(a, b)_{F} \gamma(b, \psi), a, b \in F^{\times}$. Thus, we have the commutative diagram (as in page 17 of [8])

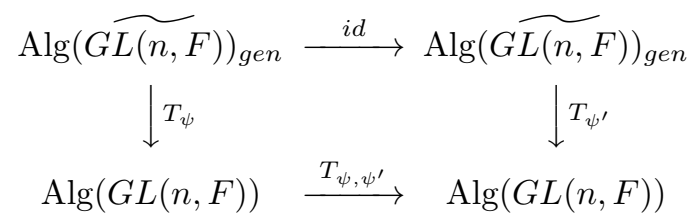

where $T_{\psi}(\rho)=\chi_{\psi}^{-1} \rho$ and $T_{\psi^{\prime}}(\rho)=\chi_{\psi^{\prime}}^{-1} \rho$ for $\rho \in \operatorname{Alg}(\widetilde{G(n, F)})_{g e n}$ and $\left(T_{\psi, \psi^{\prime}}(\pi)\right)(g)=(\operatorname{det} g, a)_{F} \pi(g)$ for $g \in G L(n, F)$ and $\pi \in \operatorname{Alg}(G L(n, F))$.

For $s=\left(n_{1}, \ldots, n_{k} ; n_{0}\right)$ and an irreducible genuine representation $\rho$ of $\widetilde{M_{s}} \leq \widetilde{S p(n, F)}$, using $\phi$ we write $\rho \stackrel{\phi}{\cong} \chi_{\psi} \pi_{1} \otimes \cdots \otimes \chi_{\psi} \pi_{k} \otimes \tau$ where $\pi_{i}$ are irreducible representations of $G L\left(n_{i}, F\right), i=1, \ldots, k$, and $\tau$ a genuine irreducible representation of $S \widetilde{p\left(n_{0}, F\right)}$. We use notation

$$
\begin{aligned}
\operatorname{Ind}_{\widetilde{M_{s}}} \widetilde{S(n, F)}(\rho) & =\operatorname{Ind}_{\widetilde{M_{s}}} \widetilde{S_{(n, F)}}\left(\chi_{\psi} \pi_{1} \otimes \cdots \otimes \chi_{\psi} \pi_{k} \otimes \tau\right) \\
& =\chi_{\psi} \pi_{1} \times \cdots \times \chi_{\psi} \pi_{k} \rtimes \tau .
\end{aligned}
$$


For an ordered partition $s=\left(n_{1}, \ldots, n_{k}\right)$ of $n$ and an irreducible genuine representation $\pi$ of $\widetilde{M}_{s} \leq \widehat{G L(n, F)}$, using $\phi$ we write $\pi \stackrel{\phi}{\cong} \chi_{\psi} \pi_{1} \otimes \cdots \otimes \chi_{\psi} \pi_{k}$ where $\pi_{i}$ are irreducible representations of $G L\left(n_{i}, F\right), i=1, \ldots, k$. We use notation

$$
\operatorname{Ind} \frac{G \widetilde{L(n, F)}}{\widetilde{M_{s}}}(\pi)=\operatorname{Ind}_{\frac{G \widetilde{L(n, F)}}{M_{s}}}\left(\chi_{\psi} \pi_{1} \otimes \cdots \otimes \chi_{\psi} \pi_{k}\right)=\chi_{\psi} \pi_{1} \times \cdots \times \chi_{\psi} \pi_{k} .
$$

Also, for an irreducible genuine representation $\tau$ of $\widetilde{G(n, F)}$ we write

$$
r_{s}(\tau)=\operatorname{Jacq} \widetilde{S p(n, F)}(\tau)
$$

Since taking Jacquet module brings representations of the cover of general linear groups in calculations, we shall need some simple facts (Propositions 4.1, 4.2 and 4.3 of [8] and Propositions 4.6, 9.1, 9.4, 9.5, 9.12 and Theorem 1.9 of $[21])$. Let ||$_{F}$ be the normalized absolute value of $F, \nu=|\operatorname{det}|_{F}$ and $\rho$ a unitary irreducible cuspidal representation of $G L(n, F)$. We denote by $\widetilde{\rho}$ its contragredient representation. Let $a, b$ be real numbers, such that $a+b+1 \in \mathbb{Z}_{>0}$. The set $\Delta=\left\{\chi_{\psi} \nu^{-b} \rho, \ldots, \chi_{\psi} \nu^{a} \rho\right\}$ is called segment.

LEMMA 2.1. The representation $\chi_{\psi} \nu^{-b} \rho \times \cdots \times \chi_{\psi} \nu^{a} \rho$ has a unique irreducible subrepresentation and a unique irreducible quotient representation. We use notation

$$
\zeta\left(-b, a, \chi_{\psi} \rho\right) \hookrightarrow \chi_{\psi} \nu^{-b} \rho \times \cdots \times \chi_{\psi} \nu^{a} \rho \rightarrow \delta\left(-b, a, \chi_{\psi} \rho\right) .
$$

Similarly

$$
\delta\left(-b, a, \chi_{\psi} \rho\right) \hookrightarrow \chi_{\psi} \nu^{a} \rho \times \cdots \times \chi_{\psi} \nu^{-b} \rho \rightarrow \zeta\left(-b, a, \chi_{\psi} \rho\right) .
$$

The representation $\delta\left(-b, a, \chi_{\psi} \rho\right)$ is the unique irreducible subquotient of $\chi_{\psi} \nu^{-b} \rho \times \cdots \times \chi_{\psi} \nu^{a} \rho$ such that $r_{(n, \ldots, n)}\left(\delta\left(-b, a, \chi_{\psi} \rho\right)\right)=\chi_{\psi} \nu^{a} \rho \otimes \cdots \otimes \chi_{\psi} \nu^{-b} \rho$. We have $\chi_{\psi}^{2} \delta\left(-\widetilde{b, a, \chi_{\psi}} \rho\right) \cong \delta\left(-a, b, \chi_{\psi} \widetilde{\rho}\right)$. For real numbers $x$ and $y$, such that $x+y+1 \in \mathbb{Z}_{\leq 0}$, we define $\delta\left(-x, y, \chi_{\psi} \rho\right)=\zeta\left(-x, y, \chi_{\psi} \rho\right)=\chi_{\psi}$ 1. The representation $\delta\left(-b_{1}, a_{1}, \chi_{\psi} \rho_{1}\right) \times \delta\left(-b_{2}, a_{2}, \chi_{\psi} \rho_{2}\right)$ reduces if and only if $\rho_{1} \cong \rho_{2}$, $a_{1}-a_{2} \in \mathbb{Z}$ and $-b_{1} \leq-b_{2}-1 \leq a_{1}<a_{2}$ or $-b_{2} \leq-b_{1}-1 \leq a_{2}<a_{1}$. In case of reducibility, the induced representation has two non-isomorphic irreducible subquotients, one of which is $\delta\left(-b_{1}, a_{2}, \chi_{\psi} \rho_{1}\right) \times \delta\left(-b_{2}, a_{1}, \chi_{\psi} \rho_{1}\right)$. In case of irreducibility we have

$$
\delta\left(-b_{1}, a_{1}, \chi_{\psi} \rho_{1}\right) \times \delta\left(-b_{2}, a_{2}, \chi_{\psi} \rho_{2}\right) \cong \delta\left(-b_{2}, a_{2}, \chi_{\psi} \rho_{2}\right) \times \delta\left(-b_{1}, a_{1}, \chi_{\psi} \rho_{1}\right) .
$$

Omitting $\chi_{\psi}$ in Lemma 2.1 gives us the notation and claim for general linear groups.

Let $R_{g e n}(\widehat{G L(n, F)})$ be the free Abelian group with the basis consisting of classes of genuine irreducible smooth representations of $\widetilde{G L(n, F)}$ and $R^{g e n}=\bigoplus_{n \geq 0} R_{g e n}(\widehat{G(n, F)})$. We have the comultiplication $m^{*}: R^{g e n} \rightarrow$ 
$R^{g e n} \otimes R^{g e n}, m^{*}(\pi)=\sum_{k=0}^{n}$ s.s. $\left(r_{(k, n-k)}(\pi)\right), \pi \in R^{g e n}$, where s.s. denotes the semisimplification. For real numbers $a$ and $b$, such that $a+b+1 \in \mathbb{Z}_{>0}$ and an irreducible cuspidal representation $\rho$ of $G L(n, F)$ we have

$$
m^{*}\left(\delta\left(-b, a, \chi_{\psi} \rho\right)\right)=\sum_{i=-b-1}^{a} \delta\left(i+1, a, \chi_{\psi} \rho\right) \otimes \delta\left(-b, i, \chi_{\psi} \rho\right) .
$$

In similar notations

$$
\begin{aligned}
m^{*} & \left(\prod_{r=1}^{s} \delta\left(-b_{r}, a_{r}, \chi_{\psi} \rho_{r}\right)\right) \\
& =\prod_{r=1}^{s}\left(\sum_{i_{r}=-b_{r}-1}^{a_{r}} \delta\left(i_{r}+1, a_{r}, \chi_{\psi} \rho_{r}\right) \otimes \delta\left(-b_{r}, i_{r}, \chi_{\psi} \rho_{r}\right)\right),
\end{aligned}
$$

where $\left(\pi_{1} \otimes \pi_{2}\right) \times\left(\pi_{3} \otimes \pi_{4}\right)=\left(\pi_{1} \times \pi_{3}\right) \otimes\left(\pi_{2} \times \pi_{4}\right)$, for $\pi_{1}, \pi_{2}, \pi_{3}, \pi_{4} \in R^{g e n}$.

Also, we let $R_{1}^{g e n}=\bigoplus_{n \geq 0} R_{g e n}(S p(n, F)), \mu^{*}=\sum_{k=0}^{n}$ s.s. $\left(r_{(k, n-k)}\right)$ : $R_{1}^{g e n} \rightarrow R^{g e n} \otimes R_{1}^{g e n}$, where $R_{\text {gen }}(\widetilde{S p(n, F)})$ denotes the free Abelian group with the basis of classes of genuine irreducible smooth representations of $\widetilde{S p(n, F)}$. In $R^{g e n}$ and $R_{1}^{g e n}$ we use relation $\pi_{2} \geq \pi_{1}$ if $\pi_{2}-\pi_{1}$ is a sum of classes of irreducible representations with positive coefficients. From Proposition 4.5 of [8], Lemma 2.1 and (2.3) we have

TheOREM 2.2. Let $\sigma$ be a genuine irreducible representation of the metaplectic group, $a, b$ real numbers such that $a+b+1 \in \mathbb{Z}_{>0}$ and $\rho$ a cuspidal irreducible representation of $G L(n, F)$. In $R_{1}^{\text {gen }}$ we have:

$$
\begin{aligned}
& \mu^{*}\left(\delta\left(-b, a, \chi_{\psi} \rho\right) \rtimes \sigma\right) \\
& =\sum_{\substack{\pi \otimes \sigma^{\prime} \leq \mu^{*}(\sigma) \\
\delta\left(-i, b, \chi_{\psi} \widetilde{\rho}\right) \times \delta\left(j+1, a, \chi_{\psi} \rho\right) \times \pi}} \sum_{\substack{j=i \\
\delta(-i}}^{a} \otimes \delta\left(i+1, j, \chi_{\psi} \rho\right) \rtimes \sigma^{\prime} .
\end{aligned}
$$

Formula (2.5), i.e. Proposition 4.5 of [8], derives from the result for classical groups, Theorems 5.4 and 6.5 of [20]. Also, by (11) of [8] and Lemma 2.1 , with notations as in Theorem 2.2 , we have in $R_{1}^{g e n}$

$$
\delta\left(-b, a, \chi_{\psi} \rho\right) \rtimes \sigma=\delta\left(-a, b, \chi_{\psi} \widetilde{\rho}\right) \rtimes \sigma .
$$

In the proof of the main theorem, we shall also need two lemmas from [19] that extend to the metaplectic group. The first lema is the metaplectic version of Lemma 3.7 of [19].

LEMMA 2.3. Let $\widetilde{P_{0}}, \widetilde{P^{\prime}}, \widetilde{P^{\prime \prime}}$ and $\widetilde{P^{\prime \prime \prime}}$ be parabolic subgroups of $\widetilde{\operatorname{Sp(n,F)}}$ with Levi factors $\widetilde{M_{0}}, \widetilde{M^{\prime}}$, $\widetilde{M^{\prime \prime}}$ and $\widetilde{M^{\prime \prime \prime}}$, such that $\widetilde{P^{\prime}} \subseteq \widetilde{P^{\prime \prime}}$ and $\widetilde{P^{\prime}} \subseteq \widetilde{P^{\prime \prime \prime}}$. 
Let $\sigma_{0}$ be an irreducible representation of $\widetilde{M}_{0}$ such that

$$
r \widetilde{S \widetilde{M^{\prime}}} \widetilde{\widetilde{\left.S^{\prime}, F\right)}}\left(\operatorname{Ind} \widetilde{S_{M_{0}}(n, F)}\left(\sigma_{0}\right)\right) \neq 0 .
$$

Assume that there exists an irreducible subquotient $\tau^{\prime \prime}$ of $\widetilde{r_{\widetilde{p(n, F)}}}\left(\operatorname{Ind}_{\widetilde{M^{\prime \prime}}} \widetilde{S p(n, F)}\left(\sigma_{0}\right)\right)$ such that for every irreducible subquotient $\tau^{\prime \prime \prime}$ of $r \widetilde{S \widetilde{S p, F)}}\left(\widetilde{M^{\prime \prime \prime}} \underset{\widetilde{S p(n, F)}}{\widetilde{M_{0}}}\left(\sigma_{0}\right)\right)$ we have:

$$
\text { s.s. }\left(r \widetilde{\bar{M}^{\prime \prime}}\left(\tau^{\prime \prime}\right)\right)+\text { s.s. }\left(r \widetilde{M^{\prime \prime \prime}}\left(\tau^{\prime \prime \prime}\right)\right) \not \leq s . s .\left(r \frac{S p(n, F)}{\widetilde{M}^{\prime}}\left(\operatorname{Ind} \widetilde{S p(n, F)}\left(\left(\sigma_{0}\right)\right)\right)\right. \text {. }
$$

Then, the induced representation $\operatorname{Ind} \frac{\widetilde{S p(n, F)}}{M_{0}}\left(\sigma_{0}\right)$ is irreducible.

Proof. First, let $\tau$ be any irreducible subquotient of $\operatorname{Ind} \widetilde{\frac{S p(n, F)}{M_{0}}}\left(\sigma_{0}\right)$. We show that $r \widetilde{S p(n, F)}(\tau) \neq 0$. Otherwise, there exist $\widetilde{M_{c}^{1}} \supsetneq \widetilde{M^{\prime}}$ and a genuine irreducible cuspidal representation $\tau_{c}^{1}$ of $\widetilde{M_{c}^{1}}$, such that $\tau_{c}^{1} \leq r \widetilde{S p(n, F)}(\tau) \leq$ $r \widetilde{S \widetilde{p(n, F)}}\left(\operatorname{Ind}_{\widetilde{M^{1}}} \widetilde{S p(n, F)}\left(\sigma_{0}\right)\right)$. By Theorem $2.4(\mathrm{~b})$ of [2], we have $\operatorname{Hom}_{\widetilde{M_{c}^{1}}}\left(r \frac{S p(n, F)}{M_{c}^{1}}\left(\operatorname{Ind}_{\widetilde{M_{0}}} \widetilde{S(n, F)}\left(\sigma_{0}\right)\right), \tau_{c}^{1}\right) \neq 0$. Frobenius reciprocity implies

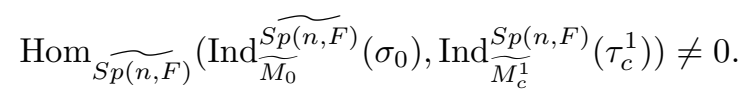

In the same way, since $r \widetilde{S \widetilde{M^{\prime}}} \widetilde{\widetilde{M^{\prime}, F}}\left(\operatorname{Ind} \widetilde{\frac{S p(n, F)}{M_{0}}}\left(\sigma_{0}\right)\right) \neq 0$, there exist $\widetilde{P_{c}^{2}} \subseteq \widetilde{P^{\prime}}$ with Levi factor $\widetilde{M_{c}^{2}}$ and a genuine irreducible cuspidal representation $\tau_{c}^{2}$ of $\widetilde{M_{c}^{2}}$ such that

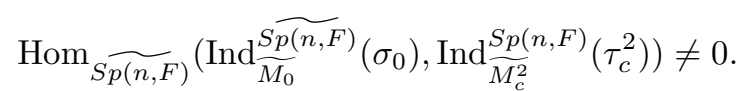

Also, there exist a parabolic subgroup $\widetilde{P_{c}}$ with Levi factor $\widetilde{M}_{c}$ and a genuine irreducible cuspidal representation $\tau_{c}$ of $\widetilde{M}_{c}$ such that $\sigma_{0} \hookrightarrow \operatorname{Ind}_{\widetilde{M_{c}}}^{\widetilde{M_{0}}}\left(\tau_{c}\right)$. Now

$$
\operatorname{Ind} \widetilde{S p(n, F)}\left(\sigma_{0}\right) \hookrightarrow \operatorname{Ind}_{\widetilde{M}_{0}} \widetilde{S p(n, F)}\left(\tau_{c}\right) .
$$

Comparing (2.7) and (2.9), Theorem 2.9 of [2] shows that $\widetilde{M_{c}^{1}}$ and $\widetilde{M}_{c}$ are associated. Also, comparing (2.8) and (2.9) shows that $\widetilde{M_{c}^{2}}$ and $\widetilde{M}_{c}$ are associated. Thus $\widetilde{M_{c}^{1}}$ and $\widetilde{M_{c}^{2}}$ are associated. But that is in contradiction with $\widetilde{M_{c}^{2}} \subseteq \widetilde{M_{c}} \subsetneq \widetilde{M_{c}^{1}}$. Thus, we proved that $r_{\widetilde{M^{\prime}}}^{\widehat{S(n, F)}}(\tau) \neq 0$. 
Now, suppose that $\operatorname{Ind} \widetilde{\widetilde{M_{0}}} \widetilde{\widetilde{S n F}}\left(\sigma_{0}\right) \geq \pi^{\prime \prime}+\pi^{\prime \prime \prime}$, where $\pi^{\prime \prime}$ and $\pi^{\prime \prime \prime}$ are irreducible subquotients and $r \widehat{\widetilde{S^{\prime \prime}(n, F)}}\left(\pi^{\prime \prime}\right) \geq \tau^{\prime \prime}$. We proved that $r \widehat{\widetilde{M^{\prime}}} \widetilde{\widetilde{M^{\prime \prime}, F}}\left(\pi^{\prime \prime \prime}\right) \neq 0$ and since $\widetilde{M^{\prime}} \subseteq \widetilde{M^{\prime \prime \prime}}$ we have $r \widetilde{S(n, F)}\left(\pi^{\prime \prime \prime}\right) \neq 0$. Let $\tau^{\prime \prime \prime}$ be an irreducible subquotient in $r_{\widetilde{M^{\prime \prime \prime}}}^{S \widetilde{(n, F)}}\left(\pi^{\prime \prime \prime}\right)$. We have

$$
\begin{aligned}
& \text { s.s. }\left(r \widetilde{M^{\prime \prime}}\left(\tau^{\prime \prime}\right)\right)+\text { s.s. }\left(r \widetilde{M^{\prime \prime \prime \prime}}\left(\tau^{\prime \prime \prime}\right)\right) \leq \text { s.s. }\left(r \widetilde{M^{\prime}} \widetilde{\bar{M}^{\prime}(n, F)}\left(\pi^{\prime \prime}\right)\right)+\text { s.s. }\left(r \widetilde{S \widetilde{p(n, F)}}\left(\pi^{\prime \prime \prime}\right)\right) \\
& \leq \text { s.s. }\left(r \widetilde{S \widetilde{p(n, F)}}\left(\operatorname{Ind} \frac{S \widetilde{S p n, F)}}{\widetilde{M_{0}}}\left(\left(\sigma_{0}\right)\right)\right)\right.
\end{aligned}
$$

which contradicts the assumption of the lemma. Thus, the representation $\operatorname{Ind} \widetilde{S_{0}} \widetilde{M_{0}(n, F)}\left(\left(\sigma_{0}\right)\right)$ is irreducible.

The second lemma is the metaplectic version of a case of Lemma 3.8 of [19] and the same proof works here, changing only group notation.

Lemma 2.4. Let $\widetilde{P_{0}}=\widetilde{M}_{0} N^{\prime}$ be a parabolic subgroup of $\widetilde{S p(n, F)}$ and $\sigma_{0}$ an irreducible unitarizable representation of the $\widetilde{M}_{0}$. If the multiplicity of $\sigma_{0}$ in $r \overline{S \frac{S(n, F)}{M_{0}}}\left(\operatorname{Ind} \frac{S \overrightarrow{p(n, F)}}{M_{0}}\left(\sigma_{0}\right)\right)$ is two, then $\operatorname{Ind} \frac{S \widetilde{S(n, F)}}{M_{0}}\left(\sigma_{0}\right)$ is either irreducible or a direct sum of two irreducible non-isomorphic representations.

Now we recall basic results related to the odd orthogonal groups. For details refer to section 1 of [10] and sections 1 and 2.1 of [9]. Let $V_{0}$ be an anisotropic quadratic space over $F$ of dimension 1 or 3 . For the classification of anisotropic quadratic spaces over $F$ one may consult page 7 of [17]. For $r \in \mathbb{Z}_{\geq 0}$, adding $r$ hyperbolic planes we obtain enlarged quadratic space which we denote by $V_{r}$. The corresponding orthogonal group is denoted by $O\left(V_{r}\right)$. Consequently, we obtained a tower of quadratic spaces and orthogonal groups. The subgroup of $O\left(V_{r}\right)$ consisting of elements of determinant one is denoted by $S O\left(V_{r}\right)$. Observe that $O\left(V_{r}\right)=S O\left(V_{r}\right) \times\{ \pm 1\}$. The orthogonal group $O\left(V_{r}\right)$ is realized as in section 1 of [10], and as there we fix a Borel subgroup. Let $m$ be an integer $0 \leq m \leq r$ and $\left(n_{1}, \ldots, n_{k}\right)$ ordered partition of $m$ if $m>0$, or $\left(n_{1}\right)=(0)$ if $m=0$. Let $s=\left(n_{1}, \ldots, n_{k} ; n_{0}\right)$ where $n_{0}=n-\left(n_{1}+\cdots+n_{k}\right)$. Such $s$ parametrize standard parabolic subgroups $P_{s}$ of $O\left(V_{r}\right)$. We have the Levi decomposition $P_{s}=M_{s} N_{s}$, where $M_{s}$ is Levi factor and $N_{s}$ unipotent radical. Here $M_{s} \cong G L\left(n_{1}, F\right) \times \cdots \times G L\left(n_{k}, F\right) \times O\left(V_{n_{0}}\right)$. Taking $P_{s}\left(S O\left(V_{r}\right)\right)=P_{s} \cap S O\left(V_{r}\right)$ we obtain standard parabolic subgroups of $S O\left(V_{r}\right)$. The Levi factor of $P_{s}\left(S O\left(V_{r}\right)\right), M_{s}\left(S O\left(V_{r}\right)\right)=M_{s} \cap S O\left(V_{r}\right)$ is isomorphic to $G L\left(n_{1}, F\right) \times \cdots \times G L\left(n_{k}, F\right) \times S O\left(V_{n_{0}}\right)$. Given smooth representations $\pi_{i}$ of $G L\left(n_{i}, F\right), i=1, \ldots, k, \pi$ of $O\left(V_{r}\right)$ and $\tau$ of $S O\left(V_{r}\right)$, we 
use the following notation for the normalized parabolic induction

$$
\begin{aligned}
& \pi_{1} \times \cdots \times \pi_{k} \rtimes \pi_{0}=\operatorname{Ind}_{M_{s}}^{O\left(V_{r}\right)}\left(\pi_{1} \otimes \cdots \otimes \pi_{k} \otimes \pi_{0}\right), \\
& \pi_{1} \times \cdots \times \pi_{k} \rtimes \tau=\operatorname{Ind}_{M_{s}\left(S O\left(V_{r}\right)\right)}^{S O\left(V_{r}\right)}\left(\pi_{1} \otimes \cdots \otimes \pi_{k} \otimes \tau\right) .
\end{aligned}
$$

Let $\rho$ be a unitary cuspidal irreducible representation of $G L(n, F)$ and $\delta(-b, a, \rho)$ the unique irreducible quotient of $\nu^{-b} \rho \times \cdots \times \nu^{a} \rho$ where $a$ and $b$ are real numbers such that $a+b$ is a nonnegative integer. Let $a \in \mathbb{N}$ and denote by $\delta(\rho, a)$ the representation $\delta\left(-\frac{a-1}{2}, \frac{a-1}{2}, \rho\right)$. We have the following theorem

THEOREM 2.5. Let $\rho_{1}, \ldots, \rho_{n}$ be irreducible unitary cuspidal representations of general linear groups $G L\left(k_{i}, F\right), k_{i} \geq 1$ and let $a_{1}, \ldots, a_{n} \in \mathbb{N}$. Suppose that $\pi$ is an irreducible square integrable representation of $S O\left(V_{r}\right)$. Then the induced representation

$$
\delta\left(\rho_{1}, a_{1}\right) \times \cdots \times \delta\left(\rho_{n}, a_{n}\right) \rtimes \pi
$$

is a multiplicity one representation of length $2^{m}$, where $m$ is a number of inequivalent representations in the set $\left\{\delta\left(\rho_{i}, a_{i}\right): i=1, \ldots, n\right\}$ such that $\delta\left(\rho_{i}, a_{i}\right) \rtimes \pi$ reduces.

Proof. This result is initially due to Goldberg, see Theorems 4.9, 4.18, 6.5 and 1.9 of [6]. The above statement is part (i) of Theorem 13.1 of [16], written without interpreting reducibility of $\delta\left(\rho_{i}, a_{i}\right) \rtimes \pi$ in terms of Jordan blocks and $L$ functions. As such it does not depend on the basic assumption (BA) given there, but we note that the article is now considered unconditional, see page 3160 of [11].

We close this section with the notion of the theta correspondance. More details can be found in section 2 of [10] and section 5 of [8]. The pair $\left(S p(n, F), O\left(V_{r}\right)\right)$ constitute a dual pair in $S p\left(n \cdot \operatorname{dim}\left(V_{r}\right), F\right)$. Let $n^{\prime}=n \cdot \operatorname{dim}\left(V_{r}\right)$. Considering preimages in $\widetilde{S p}\left(n^{\prime}, F\right), S p(n, F)$ does not split in its cover, while $O\left(V_{r}\right)$ does. Thus, given the Weil representation $\omega_{n^{\prime}, \psi}$ of $\widetilde{S p}\left(n^{\prime}, F\right)$, that depends on $\psi$, one may consider its pullback to $\widehat{S p(n, F)} \times O\left(V_{r}\right)$. We denote it by $\omega_{n, V_{r}, \psi}$. Let $\sigma$ be a genuine irreducible representation of $\widehat{S p(n, F)}$. The maximal $\sigma$-isotypic quotient of $\omega_{n, V_{r}, \psi}$ has the form $\sigma \otimes \Theta_{n, V_{r}, \psi}(\sigma)$ where $\Theta_{n, V_{r}, \psi}(\sigma)$ is a smooth representation of $O\left(V_{r}\right)$ called (big or full) theta lift of $\sigma$. If $\Theta_{n, V_{r}, \psi}(\sigma) \neq 0$, it has the unique irreducible quotient, denoted by $\theta_{n, V_{r}, \psi}(\sigma)$ and called small theta lift. Taking spaces $V_{r}$ in the same tower, let $r_{0}$ be the first index such that $\Theta_{n, V_{r_{0}}, \psi}(\sigma) \neq 0$. If $\sigma$ is cuspidal then $\Theta_{n, V_{r_{0}}, \psi}(\sigma)$ is irreducible and cuspidal (Theorem 2.1 of [10]). For an irreducible representation $\tau$ of $O\left(V_{r}\right)$ we have similar statements on the maximal $\tau$-isotypic quotient of $\omega_{n, V_{r}, \psi}$. 
Now we state some results from [4], where one can see section 2.7, Corollaries 6.2, 6.4 and 8.3 and Theorem 8.1 for more details. Because of the remark after Corollary 6.4 of [4] and Theorem 1.2 of [5], we use these results without restriction to the case when the residual caracteristic of the field $F$ is different from 2. Up to an isomorphism there are two quadratic spaces of dimension $2 n+1$ over $F$ of discriminant one. Let us denote them $V^{+}$, which has the maximal anisotropic space of dimension $n$, and $V^{-}$, which has the maximal anisotropic space of dimension $n-1$. We have:

Proposition 2.6. Let $\sigma$ be an irreducible genuine representation of $\widehat{S p(n, F)}$. Then exactly one of $\Theta_{n, V^{+}, \psi}(\sigma)$ and $\Theta_{n, V^{-}, \psi}(\sigma)$ is non-zero. Let $V$ be $V^{+}$or $V^{-}$such that $\Theta_{n, V, \psi}(\sigma)$ is non-zero. Suppose that $\sigma$ is a discrete series representation. Then the big theta lift and the small theta lift of $\sigma$ coincide, that is $\Theta_{n, V, \psi}(\sigma)=\theta_{n, V, \psi}(\sigma)$, and it is an irreducible discrete series. Denote it by $\tau$. Also let $\delta_{i}$ be discrete series representations of $G L\left(n_{i}, F\right)$, $i=1, . ., k$, where $k$ and $n_{1}, \ldots, n_{k}$ are positive integers, and let $\tau_{0}$ denote the restriction of $\tau$ on $S O(V)$. Then $\chi_{\psi} \delta_{1} \times \cdots \times \chi_{\psi} \delta_{k} \rtimes \sigma$ and $\delta_{1} \times \cdots \times \delta_{k} \rtimes \tau_{0}$ have the same number of irreducible summands, up to equivalence and ignoring multiplicities.

\section{The PROOF OF THE MAIN THEOREM}

The proof is broken in series of propositions, considered for classical groups in [19]. They cover, case by case, all posibilities for input data. In all this section $\rho$ is an irreducible unitary cuspidal representation of $G L(l, F)$ and $\sigma$ similarly genuine representation of the $\widetilde{S p(k, F)}$. We begin with the cuspidal reducibility.

Proposition 3.1. Suppose that there exists $\alpha \geq 0$ such that $\chi_{\psi} \nu^{\alpha} \rho \rtimes \sigma$ reduces. Then such $\alpha$ is a unique half-integer and $\rho \cong \widetilde{\rho}$.

Proof. Proposition 10.1 of [4] and comment below show that $\rho \cong \widetilde{\rho}$. If $\rho$ is the trivial representation of $F^{\times}$, Theorem 4.1. of [9] gives the claim. Suppose that $\rho$ is not the trivial representation of $F^{\times}$. Consider a tower of quadratic spaces $V_{r}$ and orthogonal groups $O\left(V_{r}\right)$. Recall that $\omega_{k, V_{r}, \psi}$ is the Weil representation attached to the dual pair $\widehat{S p(k, F)} \times O\left(V_{r}\right)$ and our fixed character $\psi$. Let $r_{0}$ be the smallest index such that there exists a nonzero irreducible representation $\tau$ of $O\left(V_{r_{0}}\right)$ that corresponds to $\sigma$ under the theta correspondance with respect to the Weil representation $\omega_{k, V_{r_{0}}, \psi}$. Then the representation $\tau$ is cuspidal. By Theorem 3.5 of [9] the representation $\chi_{\psi} \nu^{\alpha} \rho \rtimes \sigma$ reduces if and only if the representation $\nu^{\alpha} \rho \rtimes \tau$ of $O\left(V_{l+r_{0}}\right)$ reduces. But this representation reduces if and only if the restriction $\left(\nu^{\alpha} \rho \rtimes\right.$ $\tau)\left.\right|_{S O\left(V_{l+r_{0}}\right)} \cong \nu^{\alpha} \rho \rtimes\left(\left.\tau\right|_{S O\left(V_{r_{0}}\right)}\right)$ reduces. Now Theorem 3.1.1 of [15] implies that $\alpha \in(1 / 2) \mathbb{Z}$ and since $\alpha$ is assumed to be non-negative by Proposition 10.1 of [4] it is unique. 
We start considering situations when the cuspidal reducibility occurs.

Proposition 3.2. Suppose that there exists $\alpha>0$ such that $\chi_{\psi} \nu^{\alpha} \rho \rtimes \sigma$ reduces. Let $n \in \mathbb{Z}_{\geq 0}$. Then:

(i) The representation $\chi_{\psi} \nu^{\alpha+n} \rho \times \chi_{\psi} \nu^{\alpha+n-1} \rho \times \cdots \times \chi_{\psi} \nu^{\alpha} \rho \rtimes \sigma$ is a multiplicity one representation and contains a unique irreducible subrepresentation denoted by $\delta\left(\alpha, \alpha+n, \chi_{\psi} \rho, \sigma\right)$.

(ii) We have $\mu^{*}\left(\delta\left(\alpha, \alpha+n, \chi_{\psi} \rho, \sigma\right)\right)=\sum_{i=-1}^{n} \delta\left(\alpha+i+1, \alpha+n, \chi_{\psi} \rho\right) \otimes$ $\delta\left(\alpha, \alpha+i, \chi_{\psi} \rho, \sigma\right)$, where we take $\delta\left(\alpha, \alpha-1, \chi_{\psi} \rho, \sigma\right)=\sigma$.

(iii) The representation $\delta\left(\alpha, \alpha+n, \chi_{\psi} \rho\right) \rtimes \sigma$ reduces.

Proof. For (i), applying (2.5) multiple times on $\chi_{\psi} \nu^{\alpha+n} \rho \times \chi_{\psi} \nu^{\alpha+n-1} \rho \times$ $\cdots \times \chi_{\psi} \nu^{\alpha} \rho \rtimes \sigma$ gives in $R_{1}^{g e n}$

$$
\begin{aligned}
& r_{((n+1) l ; k)}\left(\chi_{\psi} \nu^{\alpha+n} \rho \times \chi_{\psi} \nu^{\alpha+n-1} \rho \times \cdots \times \chi_{\psi} \nu^{\alpha} \rho \rtimes \sigma\right) \\
& \quad=\sum_{\epsilon_{0}, \ldots, \epsilon_{n} \in\{ \pm 1\}} \chi_{\psi} \nu^{\epsilon_{n}(\alpha+n)} \rho \times \chi_{\psi} \nu^{\epsilon_{n-1}(\alpha+n-1)} \rho \times \cdots \times \chi_{\psi} \nu^{\epsilon_{0} \alpha} \rho \otimes \sigma .
\end{aligned}
$$

Now Proposition 2.1 of [21] implies that $\chi_{\psi} \nu^{\alpha+n} \rho \otimes \chi_{\psi} \nu^{\alpha+n-1} \rho \otimes \cdots \otimes$ $\chi_{\psi} \nu^{\alpha} \rho \otimes \sigma$, as all other subquotients, in $r_{(l, \ldots, l ; k)}\left(\chi_{\psi} \nu^{\alpha+n} \rho \times \chi_{\psi} \nu^{\alpha+n-1} \rho \times\right.$ $\left.\cdots \times \chi_{\psi} \nu^{\alpha} \rho \rtimes \sigma\right)$ appears with the multiplicity one. Thus, $\chi_{\psi} \nu^{\alpha+n} \rho \times$ $\chi_{\psi} \nu^{\alpha+n-1} \rho \times \cdots \times \chi_{\psi} \nu^{\alpha} \rho \rtimes \sigma$ is a multiplicity one representation and has a unique irreducible subrepresentation, since by Frobenius reciprocity every irreducible subrepresentation of $\chi_{\psi} \nu^{\alpha+n} \rho \times \chi_{\psi} \nu^{\alpha+n-1} \rho \times \cdots \times \chi_{\psi} \nu^{\alpha} \rho \rtimes \sigma$ has $\chi_{\psi} \nu^{\alpha+n} \rho \otimes \chi_{\psi} \nu^{\alpha+n-1} \rho \otimes \cdots \otimes \chi_{\psi} \nu^{\alpha} \rho \otimes \sigma$ as a subquotient in the Jacquet module with respect to the appropriate parabolic subgroup.

For (ii) and (iii), $\delta\left(\alpha, \alpha+n, \chi_{\psi} \rho\right) \hookrightarrow \chi_{\psi} \nu^{\alpha+n} \rho \times \chi_{\psi} \nu^{\alpha+n-1} \rho \times \cdots \times \chi_{\psi} \nu^{\alpha} \rho$ and (i) imply $\delta\left(\alpha, \alpha+n, \chi_{\psi} \rho, \sigma\right) \hookrightarrow \delta\left(\alpha, \alpha+n, \chi_{\psi} \rho\right) \rtimes \sigma$. By Theorems 3.4 and 4.6 of [12], $\delta\left(\alpha, \alpha+n, \chi_{\psi} \rho, \sigma\right)$ is a strongly positive discrete series, i.e. for each embedding of the form $\hookrightarrow \chi_{\psi} \nu^{s_{1}} \rho_{1} \times \cdots \times \chi_{\psi} \nu^{s_{m}} \rho_{m} \rtimes \sigma_{c u s p}$, where $\rho_{1}, \ldots, \rho_{m}$ are unitary irreducible cuspidal representations of $G L\left(l_{1}, F\right), \ldots, G L\left(l_{m}, F\right)$ and $\sigma_{c u s p}$ is an irreducible genuine cuspidal representation of metaplectic group, we have $s_{i}>0$ for $i=1, \ldots, m$. Now (ii) and (iii) follow from Theorem 6.1 of [13], one may also see section 7 of [14].

When the cuspidal reducibility occurs at zero we have:

Proposition 3.3. Suppose that $\chi_{\psi} \rho \rtimes \sigma$ reduces and $a, b \in \mathbb{Z}_{\geq 0}$, then the representation $\delta\left(-b, a, \chi_{\psi} \rho\right) \rtimes \sigma$ reduces.

Proof. By Proposition 3.1 we have $\widetilde{\rho} \cong \rho$. Thus $\mu^{*}\left(\chi_{\psi} \rho \rtimes \sigma\right)=\chi_{\psi} \mathbf{1} \otimes$ $\chi_{\psi} \rho \rtimes \sigma+\chi_{\psi} \rho \otimes \sigma+\chi_{\psi} \rho \otimes \sigma$. By Lemma 2.4 the representation $\chi_{\psi} \rho \rtimes \sigma$ is a direct sum of two non-isomorphic irreducible representations, say $\tau_{1}$ and $\tau_{2}$. 
We have in $R_{1}^{\text {gen }}$

$$
\begin{aligned}
\chi_{\psi} \nu^{-b} \rho \times \cdots \times \chi_{\psi} \nu^{a} \rho \rtimes \sigma \\
=\chi_{\psi} \nu^{-b} \rho \times \cdots \times \chi_{\psi} \nu^{-1} \rho \times \chi_{\psi} \nu \rho \times \cdots \times \chi_{\psi} \nu^{a} \rho \times \chi_{\psi} \rho \rtimes \sigma \\
=\chi_{\psi} \nu^{-b} \rho \times \cdots \times \chi_{\psi} \nu^{-1} \rho \times \chi_{\psi} \nu \rho \times \cdots \times \chi_{\psi} \nu^{a} \rho \rtimes \tau_{1} \\
\quad+\chi_{\psi} \nu^{-b} \rho \times \cdots \times \chi_{\psi} \nu^{-1} \rho \times \chi_{\psi} \nu \rho \times \cdots \times \chi_{\psi} \nu^{a} \rho \rtimes \tau_{2} .
\end{aligned}
$$

If we show that no Jacquet module of $\chi_{\psi} \nu^{-b} \rho \times \cdots \times \chi_{\psi} \nu^{-1} \rho \times \chi_{\psi} \nu \rho \times \cdots \times$ $\chi_{\psi} \nu^{a} \rho \rtimes \tau_{1}$ contains an irreducible subquotient of type $\pi_{2} \otimes \tau_{2}$ and that no Jacquet module of $\chi_{\psi} \nu^{-b} \rho \times \cdots \times \chi_{\psi} \nu^{-1} \rho \times \chi_{\psi} \nu \rho \times \cdots \times \chi_{\psi} \nu^{a} \rho \rtimes \tau_{2}$ contains an irreducible subquotient of type $\pi_{1} \otimes \tau_{1}$, where $\pi_{1}$ and $\pi_{2}$ are some irreducible representations, while both of these types are present in some Jacquet module of $\delta\left(-b, a, \chi_{\psi} \rho\right) \rtimes \sigma$, then we have the reducibility of $\delta\left(-b, a, \chi_{\psi} \rho\right) \rtimes \sigma$.

Suppose that there exists an irreducible representation $\pi_{2} \otimes \tau_{2} \leq$ $\mu^{*}\left(\chi_{\psi} \nu^{-b} \rho \times \cdots \times \chi_{\psi} \nu^{-1} \rho \times \chi_{\psi} \nu \rho \times \cdots \times \chi_{\psi} \nu^{a} \rho \rtimes \tau_{1}\right)$. Since $\mu^{*}\left(\tau_{1}\right)=$ $\chi_{\psi} \mathbf{1} \otimes \tau_{1}+\chi_{\psi} \rho \otimes \sigma$, applying (2.5) multiple times, there exist

$-b-1 \leq i_{-b} \leq-b, i_{-b} \leq j_{-b} \leq-b, \ldots, a-1 \leq i_{a} \leq a, i_{a} \leq j_{a} \leq a$, skipping zero index, such that either

$$
\begin{aligned}
& \pi_{2} \leq\left(\prod_{r \in\{-b, \ldots, a\} \backslash\{0\}} \delta\left(-i_{r}, r, \chi_{\psi} \rho\right) \times \delta\left(j_{r}+1, r, \chi_{\psi} \rho\right)\right), \\
& \tau_{2} \leq\left(\prod_{r \in\{-b, \ldots, a\} \backslash\{0\}} \delta\left(i_{r}+1, j_{r}, \chi_{\psi} \rho\right)\right) \rtimes \tau_{1}
\end{aligned}
$$

or

$$
\begin{aligned}
& \pi_{2} \leq \chi_{\psi} \rho \times\left(\prod_{r \in\{-b, \ldots, a\} \backslash\{0\}} \delta\left(-i_{r}, r, \chi_{\psi} \rho\right) \times \delta\left(j_{r}+1, r, \chi_{\psi} \rho\right)\right), \\
& \tau_{2} \leq\left(\prod_{r \in\{-b, \ldots, a\} \backslash\{0\}} \delta\left(i_{r}+1, j_{r}, \chi_{\psi} \rho\right)\right) \rtimes \sigma .
\end{aligned}
$$

For all $r=-b, \ldots, a ; r \neq 0$, the expression $\delta\left(i_{r}+1, j_{r}, \chi_{\psi} \rho\right)$ is either $\chi_{\psi} \nu^{r} \rho$ or omitted and since $\tau_{1} ¥ \tau_{2}$ we see that we can not find such indices. Thus, there doesn't exist an irreducible representation $\pi_{2} \otimes \tau_{2} \leq \mu^{*}\left(\chi_{\psi} \nu^{-b} \rho \times \cdots \times\right.$ $\left.\chi_{\psi} \nu^{-1} \rho \times \chi_{\psi} \nu \rho \times \cdots \times \chi_{\psi} \nu^{a} \rho \rtimes \tau_{1}\right)$. In the same way, there doesn't exist an irreducible representation $\pi_{1} \otimes \tau_{1} \leq \mu^{*}\left(\chi_{\psi} \nu^{-b} \rho \times \cdots \times \chi_{\psi} \nu^{-1} \rho \times \chi_{\psi} \nu \rho \times \cdots \times\right.$ 
$\left.\chi_{\psi} \nu^{a} \rho \rtimes \tau_{2}\right)$. On the other hand

$$
\begin{aligned}
& \mu^{*}\left(\delta\left(-b, a, \chi_{\psi} \rho\right) \rtimes \sigma\right) \\
& \quad=\sum_{i=-b-1}^{a} \sum_{j=i}^{a} \delta\left(-i, b, \chi_{\psi} \rho\right) \times \delta\left(j+1, a, \chi_{\psi} \rho\right) \times \pi \otimes \delta\left(i+1, j, \chi_{\psi} \rho\right) \rtimes \sigma^{\prime}
\end{aligned}
$$

contains (for $i=-1, j=0$ )

$$
\begin{aligned}
& \delta\left(1, b, \chi_{\psi} \rho\right) \times \delta\left(1, a, \chi_{\psi} \rho\right) \otimes \chi_{\psi} \rho \rtimes \sigma \\
& \quad=\delta\left(1, b, \chi_{\psi} \rho\right) \times \delta\left(1, a, \chi_{\psi} \rho\right) \otimes \tau_{1}+\delta\left(1, b, \chi_{\psi} \rho\right) \times \delta\left(1, a, \chi_{\psi} \rho\right) \otimes \tau_{2} .
\end{aligned}
$$

Thus the representation $\delta\left(-b, a, \chi_{\psi} \rho\right) \rtimes \sigma$ reduces.

Now we cover remaining situations when the cuspidal reducibility occurs.

Proposition 3.4. Let $\alpha>0$ be such that $\chi_{\psi} \nu^{\alpha} \rtimes \sigma$ reduces and $m, n \in$ $\mathbb{Z}_{\geq 0}$. Then the representation $\delta\left(\alpha-m, \alpha+n, \chi_{\psi} \rho\right) \rtimes \sigma$ reduces.

Proof. We may suppose that $|\alpha-m| \leq \alpha+n$. Also, because of Proposition 3.2 , assume that $m \geq 1$. Observe that

$$
\begin{gathered}
\delta\left(\alpha-m, \alpha+n, \chi_{\psi} \rho\right) \rtimes \sigma \leq \delta\left(\alpha-m, \alpha-1, \chi_{\psi} \rho\right) \times \delta\left(\alpha, \alpha+n, \chi_{\psi} \rho\right) \rtimes \sigma, \\
\delta\left(\alpha-m, \alpha-1, \chi_{\psi} \rho\right) \times \delta\left(\alpha, \alpha+n, \chi_{\psi} \rho, \sigma\right) \\
\leq \delta\left(\alpha-m, \alpha-1, \chi_{\psi} \rho\right) \times \delta\left(\alpha, \alpha+n, \chi_{\psi} \rho\right) \rtimes \sigma .
\end{gathered}
$$

Looking at Jacquet modules, one shows, as in Theorem 13.2 of [19], that all representations in (3.2) have common irreducible subquotient, appearing with the multiplicity one, but also

$$
\delta\left(\alpha-m, \alpha+n, \chi_{\psi} \rho\right) \rtimes \sigma \not \leq \delta\left(\alpha-m, \alpha-1, \chi_{\psi} \rho\right) \times \delta\left(\alpha, \alpha+n, \chi_{\psi} \rho, \sigma\right),
$$

which implies reducibility of the representation $\delta\left(\alpha-m, \alpha+n, \chi_{\psi} \rho\right) \rtimes \sigma$.

From now on we consider situations when the cuspidal reducibility doesn't occur. We start with the case when $\rho$ is not self-dual.

Proposition 3.5. Suppose $\rho \nsubseteq \widetilde{\rho}$. Let $a, b$ be real numbers such that $a+b+1 \in \mathbb{Z}_{>0}$. Then the representation $\delta\left(-b, a, \chi_{\psi} \rho\right) \rtimes \sigma$ is irreducible.

Proof. Let $n \in \mathbb{Z}_{\geq 0}$. By an induction we prove irreducibility of the representation $\pi=\delta\left(-b,-b+n, \chi_{\psi} \rho\right) \rtimes \sigma$. Case $n=0$ is covered by Proposition 3.1. Assume $n \geq 1$ and that the statement is valid for strictly less non negative integers. By (2.5) we have

$\mu^{*}(\pi)=\sum_{i=-b-1}^{-b+n} \sum_{j=i}^{-b+n} \delta\left(-i, b, \chi_{\psi} \widetilde{\rho}\right) \times \delta\left(j+1,-b+n, \chi_{\psi} \rho\right) \otimes \delta\left(i+1, j, \chi_{\psi} \rho\right) \rtimes \sigma$. 
Thus

$$
\begin{aligned}
\text { s.s. }( & \left.r_{(l ; n l+k)}(\pi)\right) \\
= & \chi_{\psi} \nu^{b} \widetilde{\rho} \otimes \delta\left(-b+1,-b+n, \chi_{\psi} \rho\right) \rtimes \sigma \\
& +\chi_{\psi} \nu^{-b+n} \rho \otimes \delta\left(-b,-b+n-1, \chi_{\psi} \rho\right) \rtimes \sigma,
\end{aligned}
$$

$$
\text { s.s. }\left(r_{((n+1) l ; k)}(\pi)\right)=\sum_{i=-b-1}^{-b+n} \delta\left(-i, b, \chi_{\psi} \widetilde{\rho}\right) \times \delta\left(i+1,-b+n, \chi_{\psi} \rho\right) \otimes \sigma .
$$

We use Lemma 2.3. Let $\tau^{\prime \prime}=\chi_{\psi} \nu^{b} \widetilde{\rho} \times \delta\left(-b+1,-b+n, \chi_{\psi} \rho\right) \otimes \sigma$, an irreducible sumand for $i=-b$ in (3.4). Sumands on the right hand side of (3.3) are irreducible by the assumption of the induction. Take $\tau^{\prime \prime \prime}=\chi_{\psi} \nu^{b} \widetilde{\rho} \otimes \delta(-b+$ $\left.1,-b+n, \chi_{\psi} \rho\right) \rtimes \sigma$. Let id denote the identity map. Suppose that

$(3.5)\left(\mathrm{id} \otimes\right.$ s.s. $\left.\left(r_{(l, \ldots, l ; k)}\right)\right)\left(\tau^{\prime \prime \prime}\right)+\left(\right.$ s.s. $\left.\left.\left(r_{(l, \ldots, l)}\right)\right) \otimes \mathrm{id}\right)\left(\tau^{\prime \prime}\right) \leq$ s.s. $\left(r_{(l, \ldots, l ; k)}(\pi)\right)$.

Because of (3.3) we have

(s.s. $\left.\left(r_{(l, \ldots, l)}\right) \otimes \mathrm{id}\right)\left(\tau^{\prime \prime}\right) \leq \chi_{\psi} \nu^{-b+n} \rho \otimes$ s.s. $\left(r_{(l, \ldots, l ; k)}\left(\delta\left(-b,-b+n-1, \chi_{\psi} \rho\right) \rtimes \sigma\right)\right)$, a contradiction. On the other hand if we take $\tau^{\prime \prime \prime}=\chi_{\psi} \nu^{-b+n} \rho \otimes \delta(-b,-b+$ $\left.n-1, \chi_{\psi} \rho\right) \rtimes \sigma$ assuming (3.5) implies (s.s. $\left.\left(r_{(l, \ldots, l)}\right) \otimes \mathrm{id}\right)\left(\tau^{\prime \prime}\right) \leq \chi_{\psi} \nu^{b} \widetilde{\rho} \otimes$ s.s. $\left(r_{(l, \ldots, l ; k)}(\delta(-b+1,-b+n) \rtimes \sigma)\right)$ which is again not valid. Now Lemma 2.3 implies irreducibility of $\pi$.

Next two propositions consider irreducibility when $\delta\left(-b, a, \chi_{\psi} \rho\right)$ is unitary and the cuspidal reducibility does not occur. They are the metaplectic version of Propositions 4.1 and 4.2 of [19] and can be proved in the same way.

Proposition 3.6. Let $n \in \mathbb{Z}_{\geq 0}$ and suppose that $\chi_{\psi} \nu^{i} \rho \rtimes \sigma$ is irreducible for all $-n-\frac{1}{2} \leq i \leq n+\frac{1}{2}, i \in \frac{1}{2}+\mathbb{Z}$. Then the representation $\delta\left(-n-\frac{1}{2}, n+\right.$ $\left.\frac{1}{2}, \chi_{\psi} \rho\right) \rtimes \sigma$ is irreducible.

Proposition 3.7. Let $n \in \mathbb{Z}_{\geq 0}$ and suppose that $\chi_{\psi} \nu^{i} \rho \rtimes \sigma$ is irreducible for all $-n \leq i \leq n, i \in \mathbb{Z}$. Then the representation $\delta\left(-n, n, \chi_{\psi} \rho\right) \rtimes \sigma$ is irreducible.

Now we examine remaining situations when the cuspidal reducibility does not occur.

LEMMA 3.8. Suppose that representations $\chi_{\psi} \rho \rtimes \sigma$ and $\chi_{\psi} \nu \rho \rtimes \sigma$ are irreducible, then the representation $\delta\left(0,1, \chi_{\psi} \rho\right) \rtimes \sigma$ is irreducible.

Proof. Because of Proposition 3.5 we may assume that $\widetilde{\rho} \cong \rho$. Now we only need to prove that all possible irreducible subquotients of $\chi_{\psi} \nu \rho \times$ $\delta\left(-1,1, \chi_{\psi} \rho\right) \rtimes \sigma$ are mutually isomorphic and the rest of the proof is the same as in Lemma A.4. of [3]. Let $\tau$ be the theta lift of $\sigma$ as in Proposition 2.6. Using the notation as in Proposition 2.6, it is an irreducible discrete series 
of $O(V)$ and $\tau_{0}$ denotes its restriction to $S O(V)$. The same proposition implies that $\chi_{\psi} \nu \rho \times \delta\left(-1,1, \chi_{\psi} \rho\right) \rtimes \sigma$ and $\nu \rho \times \delta(-1,1, \rho) \rtimes \tau_{0}$ have the same number of irreducible summands, up to equivalence and ignoring multiplicities. Also $\delta\left(-1,1, \chi_{\psi} \rho\right) \rtimes \sigma$ and $\delta(-1,1, \rho) \rtimes \tau_{0}$ have the same number of irreducible summands, up to equivalence and ignoring multiplicities. By Proposition 3.7 the representation $\delta\left(-1,1, \chi_{\psi} \rho\right) \rtimes \sigma$ is irreducible. Theorem 2.5 implies that $\delta(-1,1, \rho) \rtimes \tau_{0}$ is irreducible. Similarly $\rho \rtimes \tau_{0}$ is irreducible. Now Theorem 2.5 implies that $\rho \times \delta(-1,1, \rho) \rtimes \tau_{0}$ is irreducible. By Proposition 2.6 all possible irreducible subquotients of $\chi_{\psi} \nu \rho \times \delta\left(-1,1, \chi_{\psi} \rho\right) \rtimes \sigma$ are mutually isomorphic and the proof is finished. We note that another proof of the lemma is given in Proposition 3.16 of [7].

Proposition 3.9. Let $b \in \mathbb{R}$ and $n \in \mathbb{Z}_{\geq 0}$. Suppose that $\chi_{\psi} \nu^{i} \rho \rtimes \sigma$ is irreducible for all $-b \leq i \leq-b+n$ where $-b-i \in \mathbb{Z}$. Then the representation $\pi=\delta\left(-b,-b+n, \chi_{\psi} \rho\right) \rtimes \sigma$ is irreducible.

Proof. First, because of Proposition 3.5, we may assume that $\rho$ is selfdual. Proof goes by the induction over $n$. Case $n=0$ holds. Let $n>0$ and assume that the statement is valid for non-negative integers strictly less than $n$. Using (2.5), we obtain

$$
\begin{gathered}
\text { s.s. }\left(r_{(l ; n l+k)}(\pi)\right) \\
=\chi_{\psi} \nu^{-b+n} \rho \otimes \delta\left(-b,-b+n-1, \chi_{\psi} \rho\right) \rtimes \sigma \\
\quad+\chi_{\psi} \nu^{b} \rho \otimes \delta\left(-b+1,-b+n, \chi_{\psi} \rho\right) \rtimes \sigma, \\
\text { s.s. }\left(r_{((n+1) l ; k)}(\pi)\right)=\sum_{i=-b-1}^{-b+n} \delta\left(-i, b, \chi_{\psi} \rho\right) \times \delta\left(i+1,-b+n, \chi_{\psi} \rho\right) \otimes \sigma .
\end{gathered}
$$

By the induction hypothesis, both sumands in (3.6) are irreducible. Now we have two cases.

The first case is $\beta \neq 0$. Because of Propositions 3.6 and 3.7 we may assume that $-(-b) \neq-b+n$. Also, because of (2.6) we may assume that $(-b+(-b+n)) / 2>0$. This implies irreducibility of $\tau^{\prime \prime}=\chi_{\psi} \nu^{b} \rho \times \delta(-b+$ $\left.1,-b+n, \chi_{\psi} \rho\right) \otimes \sigma$, a sumand in (3.7) for $i=-b$. We want to use Lemma 2.3. So first take $\tau^{\prime \prime \prime}=\chi_{\psi} \nu^{-b+n} \rho \otimes \delta\left(-b,-b+n-1, \chi_{\psi} \rho\right) \rtimes \sigma$ and assume

$(3.8)\left(\mathrm{id} \otimes\right.$ s.s. $\left.\left(r_{(l, \ldots, l ; k)}\right)\right)\left(\tau^{\prime \prime \prime}\right)+\left(\right.$ s.s. $\left.\left(r_{(l, \ldots, l)}\right) \otimes \mathrm{id}\right)\left(\tau^{\prime \prime}\right) \leq\left(\right.$ s.s. $\left.\left(r_{(l, \ldots, l ; k)}\right)\right)(\pi)$.

Then (3.6) implies (s.s. $\left.\left(r_{(l, \ldots, l)}\right) \otimes \mathrm{id}\right)\left(\tau^{\prime \prime}\right) \leq \chi_{\psi} \nu^{b} \rho \otimes$ (s.s. $\left.\left(r_{(l, \ldots, l ; k)}\right)\right)(\delta(-b+$ $\left.\left.1,-b+n, \chi_{\psi} \rho\right) \rtimes \sigma\right)$, a contradiction. Now take $\tau^{\prime \prime \prime}=\chi_{\psi} \nu^{b} \rho \otimes \delta(-b+1,-b+$ $\left.n, \chi_{\psi} \rho\right) \rtimes \sigma$ and assume (3.8). The equation (3.6) implies (s.s. $\left(r_{(l, \ldots, l)}\right) \otimes$ id) $\left(\tau^{\prime \prime}\right) \leq \chi_{\psi} \nu^{-b+n} \rho \otimes$ (s.s. $\left.\left(r_{(l, \ldots, l ; k)}\right)\right)\left(\delta\left(-b,-b+n-1, \chi_{\psi} \rho\right) \rtimes \sigma\right)$, again contradiction, and Lemma 2.3 implies irreducibility of the representation $\pi=\delta\left(-\beta,-\beta+n, \chi_{\psi} \rho\right) \rtimes \sigma$. 
The second case is $\beta=0$. We first observe that the statement is valid for $n=1$. That is, by Lemma 3.8 if $\chi_{\psi} \rho \rtimes \sigma$ and $\chi_{\psi} \nu \rho \rtimes \sigma$ are irreducible, then the representation $\delta\left(0,1, \chi_{\psi} \rho\right) \rtimes \sigma$ is irreducible. Thus we may assume $n \geq 2$. Again, we use Lemma 2.3. Take $\tau^{\prime \prime}=\delta\left(-1,0, \chi_{\psi} \rho\right) \times \delta\left(2, n, \chi_{\psi} \rho\right) \otimes$ $\sigma$, an irreducible sumand in (3.7) for $i=1$, and first let $\tau^{\prime \prime \prime}=\chi_{\psi} \nu^{n} \rho \otimes$ $\delta\left(0, n-1, \chi_{\psi} \rho\right) \rtimes \sigma$. Assuming (3.8), the equation (3.6) implies $\left(\right.$ s.s. $\left(r_{(l, \ldots, l)}\right) \otimes$ id) $\left(\tau^{\prime \prime}\right) \leq \chi_{\psi} \rho \otimes\left(\right.$ s.s. $\left.\left(r_{(l, \ldots, l ; k)}\right)\right)\left(\delta\left(1, n, \chi_{\psi} \rho\right) \rtimes \sigma\right)$, a contradiction. Now take $\tau^{\prime \prime \prime}=\chi_{\psi} \rho \otimes \delta\left(1, n, \chi_{\psi} \rho\right) \rtimes \sigma$ and assume (3.8). The equation (3.6) implies $\left(\right.$ s.s. $\left.\left(r_{(l, \ldots, l)}\right) \otimes \mathrm{id}\right)\left(\tau^{\prime \prime}\right) \leq \chi_{\psi} \nu^{n} \rho \otimes\left(\right.$ s.s. $\left.\left(r_{(l, \ldots, l ; k)}\right)\right)\left(\delta\left(0, n-1, \chi_{\psi} \rho\right) \rtimes \sigma\right)$, again contradiction, and Lemma 2.3 implies irreducibility of the representation $\pi=$ $\delta\left(0, n, \chi_{\psi} \rho\right) \rtimes \sigma$.

This concludes the proof of the proposition.

The proof of the main theorem is complete.

\section{REFERENCES}

[1] I. N. Bernšteı̆n and A. V. Zelevinskiǔ, Representations of the group $G L(n, F)$, where $F$ is a local non-Archimedean field, Uspehi Mat. Nauk 31 (1976), 5-70.

[2] I. N. Bernstein and A. V. Zelevinsky, Induced representations of reductive p-adic groups. I, Ann. Sci. École Norm. Sup. (4) 10 (1977), 441-472.

[3] I. Ciganović and N. Grbac, The Zelevinsky classification of unramified representations of the metaplectic group, J. Algebra 454 (2016), 357-399.

[4] W. T. Gan and G. Savin, Representations of metaplectic groups I: epsilon dichotomy and local Langlands correspondence, Compos. Math. 148 (2012), 1655-1694.

[5] W. T. Gan and S. Takeda, A proof of the Howe duality conjecture, J. Amer. Math. Soc. 29 (2016), 473-493.

[6] D. Goldberg, Reducibility of induced representation for $S p(2 n)$ and $S O(n)$, Amer. J. Math. 116 (1994), 1101-1151.

[7] M. Hanzer and I. Matić, The unitary dual of p-adic $\widetilde{\operatorname{Sp}(2)}$, Pacific J. Math. 248 (2010), $107-137$.

[8] M. Hanzer and G. Muić, Parabolic induction and Jacquet functors for metaplectic groups, J. Algebra 323 (2010), 241-260.

[9] M. Hanzer and G. Muić, Rank one reducibility for metaplectic groups via theta correspondence, Canad. J. Math. 63 (2011), 591-615.

[10] S. S. Kudla, On the local theta-correspondence, Invent. Math. 83 (1986), 229-255.

[11] I. Matić, First occurrence indices of tempered representations of metaplectic groups, Proc. Amer. Math. Soc. 144 (2016), 3157-3172.

[12] I. Matić, Strongly positive representations of metaplectic groups, J. Algebra 334 (2011), $255-274$.

[13] I. Matić, Jacquet modules of strongly positive representations of the metaplectic group $\widetilde{S p(n)}$, Trans. Amer. Math. Soc. 365 (2013), 2755-2778.

[14] I. Matić and M. Tadić, On Jacquet modules of representations of segment type, Manuscripta Math. 147 (2015), 437-476.

[15] C. Møglin, Paquetes stables des séries discrètes accessibles par endoscopie tordue; leur paramètre de Langlands, in Automorphic forms and related geometry: assessing the Legacy of I. I. Piatetski-Shapiro, Contemp. Math. 614, Amer. Math. Soc., Providence, 2014, 295-336. 
[16] C. Mœglin and M. Tadić, Construction of discrete series for classical p-adic groups, J. Amer. Math. Soc. 15 (2002), 715-786.

[17] C. Mœglin, M.-F. Vignéras and J.-L. Waldspurger, Correspondances de Howe sur un corps p-adique, Lecture Notes in Mathematics 1291, Springer-Verlag, Berlin, 1987.

[18] R. Ranga Rao, On some explicit formulas in the theory of Weil representation, Pacific J. Math. 157 (1993), 335-371.

[19] M. Tadić, On reducibility of parabolic induction, Israel J. Math. 107 (1998), 29-91.

[20] M. Tadić, Structure arising from induction and Jacquet modules of representations of classical p-adic groups, J. Algebra 177 (1995), 1-33.

[21] A. V. Zelevinsky, Induced representations of reductive p-adic groups. II. On irreducible representations of $G L(n)$, Ann. Sci. École Norm. Sup. (4) 13 (1980), 165-210.

I. Ciganović

Department of Mathematics

University of Zagreb

10000 Zagreb

Croatia

E-mail: igor.ciganovic@math.hr

Received: 8.8.2015.

Revised: 17.12.2016. \& 20.2.2017. 